\title{
The Reduction of RI, a Novel Repressor Protein for Monoamine Oxidase A, in Major Depressive Disorder
}

\author{
Shakevia Johnson', Craig A Stockmeier ${ }^{1,2}$, Jeffrey H Meyer ${ }^{3}$, Mark C Austin', Paul R Albert ${ }^{4}$, Junming Wang ${ }^{5}$, \\ Warren L May ${ }^{6}$, Grazyna Rajkowska', James C Overholser ${ }^{7}$, George Jurjus $^{2}$, Lesa Dieter ${ }^{7}$, Chandra Johnson ', \\ Donald B Sittman ${ }^{8}$ and Xiao-Ming Ou,
}

'Department of Psychiatry and Human Behavior, University of Mississippi Medical Center, Jackson, MS, USA; ${ }^{2}$ Department of Psychiatry, Case Western Reserve University, Cleveland, OH, USA; ${ }^{3}$ Department of Psychiatry and Centre for Addiction and Mental Health, University of Toronto, Toronto, ON, Canada; ${ }^{4}$ Department of Neuroscience, Ottawa Hospital Research Institute, Ottawa, ON, Canada; ${ }^{5}$ Department of Pathology, University of Mississippi Medical Center, Jackson, MS, USA; ${ }^{6}$ Biostatistics Center, University of Mississippi Medical Center, Jackson, MS, USA; ${ }^{7}$ Department of Psychology, Case Western Reserve University, Cleveland, OH, USA; ${ }^{8}$ Department of Biochemistry, University of Mississippi Medical Center, Jackson, MS, USA

\begin{abstract}
The novel transcriptional repressor protein, RI (JPO2/CDCA7L/RAM2), inhibits monoamine oxidase A (MAO A) gene expression and influences cell proliferation and survival. MAO A is implicated in several neuropsychiatric illnesses and highly elevated in major depressive disorder (MDD); however, whether RI is involved in these disorders is unknown. This study evaluates the role of RI in depressed subjects either untreated or treated with antidepressant drugs. RI protein levels were determined in the postmortem prefrontal cortex of 18 untreated MDD subjects and 12 medicated MDD subjects compared with 18 matched psychiatrically normal control subjects. Western blot analysis showed that RI was significantly decreased by $37.5 \%(p<0.005)$ in untreated MDD subjects. The RI level in medicated MDD subjects was also significantly lower (by 30\%; $p<0.05$ ) compared with control subjects, but was not significantly different compared with untreated MDD subjects. Interestingly, the reduction in RI was significantly correlated with an increase (approximately 40\%; $p<0.05$ ) in MAO A protein levels within the MDD groups compared with controls. Consistent with the change in MAO A protein expression, the MAO A catalytic activity was significantly greater in both MDD groups compared with controls. These results suggest that reduced $\mathrm{RI}$ may lead to elevated MAO A levels in untreated and treated MDD subjects; moreover, the reduction of $\mathrm{RI}$ has been implicated in apoptotic cell death and apoptosis has also been observed in the brains of MDD subjects. Therefore, modulation of RI levels may provide a new therapeutic target in the development of more effective strategies to treat MDD. Neuropsychopharmacology (201 I) 36, 2 139-2148; doi:10.1038/npp.201 I.105; published online 8 June 201 I
\end{abstract}

Keywords: major depressive disorder; transcriptional repressor protein; monoamine oxidase; treatment; selective serotonin reuptake inhibitor; prefrontal cortex

\section{INTRODUCTION}

As a worldwide health burden, major depressive disorder (MDD) is widely distributed among the population and has a range of symptom severity and results in cognitive, social, professional, and economic impairment. In the United States, the treatment for MDD has a high recurrence rate; $50 \%$ of MDD patients are expected to have a subsequent depressive episode within 2 years (Keller and Boland, 1998) and $51.1 \%$ of functional disability cases are due solely to

*Correspondence: Dr X-M Ou, Department of Psychiatry and Human Behavior (G-109), University of Mississippi Medical Center, $2500 \mathrm{~N}$. State Street, Jackson, MS 39216, USA, Tel: + I 601 984 5893, Fax: + | 601984 5899, E-mail: xou@umc.edu

Received 4 February 201।; revised 5 May 20 II; accepted 5 May 201 I
MDD (Egede, 2004). A highly significant increase in monoamine oxidase A (MAO A; an enzyme that degrades monoamine neurotransmitters) has been discovered in patients with MDD by positron emission tomography (PET) (Meyer et al, 2006; Meyer et al, 2009). Since MAO A metabolizes monoamines required for normative mood, such as serotonin, norepinephrine, and dopamine (Chouinard et al, 1985; Reilly et al, 1997; Young et al, 1985; Morilak and Frazer, 2004), elevated MAO A is viewed as a pathological process in depressive disorders and in those who are at risk for MDD (Meyer et al, 2006; Meyer et al, 2009; Sacher et al, 2010).

MAO can be differentiated into two types: MAO A and MAO B; however, evidence shows that MAO A is more often implicated in mental disorders (Cases et al, 1995; Shih and Chen, 1999; Youdim et al, 2006), including depressive 
disorders (Du et al, 2004; Du et al, 2002; Fan et al, 2010; Sacher et al, 2010). While one problematic implication for elevated MAO A levels is excessive catalysis of neurotransmitters (Goridis and Neff, 1971), a second problematic implication for elevated MAO $\mathrm{A}$ is that as MAO A degrades these neurotransmitters, reactive oxygen species such as hydrogen peroxide $\left(\mathrm{H}_{2} \mathrm{O}_{2}\right)$ are produced, and these reactive oxygen species may lead to apoptotic cell death (De Zutter and Davis, 2001; Ou et al, 2009b; Youdim and Bakhle, 2006; Youdim et al, 2004). In vitro studies suggest that mitochondrial dysfunction and apoptotic cell death may be induced by MAO-dependent $\mathrm{H}_{2} \mathrm{O}_{2}$ production (Kunduzova et al, 2002). Importantly, signs of cell death and oxidative damage have been observed in the brains of depressed patients (Andreazza et al, 2010; Rajkowska et al, 2005; Schmidt and Duman, 2007), including patients with MDD (Duman and Monteggia, 2006; Dwivedi et al, 2009; Gawryluk et al, 2010; Kang et al, 2007). Hence, the increased expression of MAO A may participate in the apoptotic process seen in MDD, in addition to its primary monoamine-lowering activity that occurs during MDD.

MAO A is located on the X chromosome (Xp11.23Xp22.1) (Shih, 1991), where the core promoter region consists of two 90-base pair repeats with Sp1-binding elements (Shih et al, 1993; Zhu et al, 1994). R1 (JPO2/ CDCA7L/RAM2), a 454-amino-acid protein that binds directly to the MAO A promoter in vivo, has been identified as an upstream, transcriptional repressor of MAO A (Chen et al, 2005). R1-transfected neuroblastoma cells (SK-N-BE (2)-C) yield an inhibition of the MAO A promoter and enzymatic activity. The inhibition of MAO A afforded by R1 parallels the level of $\mathrm{R} 1$ protein expression (Chen et al, 2005). Regulation of MAO A expression by R1 occurs at the Sp1-binding sites of the MAO A promoter (Chen et al, 2005), resulting in decreased cellular death rates. Furthermore, R1 (also known as JPO2) is a c-Myc-binding protein that serves as a nuclear transcription factor (Huang et al, 2005). R1, which is widely present in the human brain and other tissues, has also been implicated in the downregulation of MAO B (Chen et al, 2011) and in the inhibition of human immunodeficiency virus-1 replication (Bartholomeeusen et al, 2007; Maertens et al, 2006). While over-expression of R1 in cultured cells decreases levels of MAO A protein, it also increases cell proliferation, thereby indicating a beneficial role for $\mathrm{R} 1$ in the promotion of cell growth (Ou et al, 2006b). On the other hand, R1-knockdown in cultured cells decreases cell proliferation but increases MAO A and caspase-3, a cell death protein, suggesting that reduced $\mathrm{R} 1$ may contribute to MAO A-mediated cell death (Ou et al, 2006b).

We hypothesize that the MAO A-repressor protein, R1, may be reduced in postmortem brain tissue from subjects with MDD as increased MAO A levels have been implicated in the pathogenesis of MDD (Meyer et al, 2006; Meyer et al, 2009; Sherif et al, 1991). A second reason why we hypothesize that decreased R1 levels occur in MDD is because lower $\mathrm{R} 1$ levels have been implicated in reduced cell proliferation and increased apoptotic cell death (Ou et al, 2006b), and there is evidence for the latter in MDD (Duman and Monteggia, 2006; Dwivedi et al, 2009; Gawryluk et al, 2010; Kang et al, 2007); however, R1 protein expression has not been previously evaluated in human postmortem brains of subjects with MDD. This study will constitute the initial evaluation of R1 protein levels in human postmortem brain tissue related to the molecular basis underlying the pathology of MDD.

\section{MATERIALS AND METHODS}

\section{Human Subjects and Tissue Collection}

Brain samples (Brodmann area 8/9; right hemisphere) were collected at autopsy at the Cuyahoga County Coroner's Office (Cleveland, Ohio) after acquiring informed written consent from the legally defined next-of-kin of all subjects (Ou et al, 2009b; Stockmeier et al, 2009). Knowledgeable informants for all subjects were interviewed and retrospective psychiatric assessments were conducted in accordance with the declaration of Helsinki (Stockmeier et al, 2009) and the Institutional Review Board policies at University Hospitals of Cleveland and the University of Mississippi Medical Center. Research on informant-based assessment of Axis I disorders has revealed that diagnoses from structured clinical interviews with family members are in agreement with diagnoses based on reviewing the subject's medical records or interviewing the subject (Deep-Soboslay et al, 2005; Dejong and Overholser, 2009; Kelly and Mann, 1996). A trained interviewer administered the structured clinical interview for DSM-IV psychiatric disorders to knowledgeable next-of-kin of the subjects to determine current and lifetime Axis I psychopathology (First et al, 1996).

There are three groups in this study. Eighteen subjects met the DSM-IV criteria for MDD (American Psychiatric Association) and 18 psychiatrically normal control subjects. An additional group (12 subjects) also met the DSM-IV criteria for MDD; however, they were actively being treated with antidepressant drugs (ADs) at the time of their deaths. Demographic and clinical characteristics for all three groups are listed in Table 1.

Eighteen (18) psychiatrically normal control subjects. For the psychiatrically normal control subjects, the average age (years, mean \pm SEM) was $49.6 \pm 3.4$ and 11 subjects were male (Table 1). Age, gender, race, smoking status, postmortem interval (PMI), $\mathrm{pH}$, freezer storage time, prescribed medication, and toxicology reports are disclosed for each healthy control subject (Supplementary Table 1a). These healthy control subjects had postmortem toxicology screenings that were negative for the presence of ADs. The cause of death for the control subjects included cardiovascular disease, bronchopneumonia, myocardial infarction, carbon monoxide poisoning, blunt trauma, and homicide (data not shown).

Eighteen (18) untreated subjects with MDD. MDD subjects and psychiatrically normal controls were matched as closely as possible (Supplementary Table 1b). The average age (years, mean \pm SEM) for subjects with MDD was $54.6 \pm 4.7$ and 12 MDD subjects were male (Table 1). Twelve subjects were victims of suicide (67\%); other causes of death for this group included cardiovascular disease, homicide, and chronic asthma (data not shown).

Thirteen untreated MDD subjects had no history (inpatient or outpatient) of psychoactive drug treatment 
Table I Demographical and Clinical Characteristics Data of Psychiatrically Normal Control (Healthy) Subjects, MDD Subjects, and MDD Subjects with Antidepressant Drugs (AD)

\begin{tabular}{|c|c|c|c|}
\hline & Healthy $(n=18)$ & MDD, no AD $(n=18)$ & MDD, with AD $(n=12)$ \\
\hline Age, year, mean (SEM) & $49.6(3.4)$ & $54.6(4.7)$ & $55.3(5.7)$ \\
\hline Male, number (\%) & II (6I.I) & $12(66.7)$ & $8(66.7)$ \\
\hline Female, number (\%) & $7(38.9)$ & $6(33.3)$ & $4(33.3)$ \\
\hline \multicolumn{4}{|l|}{ Race, number (\%) } \\
\hline African American & $8(44.4)$ & $5(27.8)$ & । (8.3) \\
\hline PMI (h), mean (SEM) & $19.8(2.0)$ & $20.7(1.6)$ & $21.0(2.1)$ \\
\hline Tissue $\mathrm{pH}$, mean (SEM) & $6.6(0.01)$ & $6.6(0.1)$ & $6.6(0.1)$ \\
\hline Storage time in freezer (year), mean (SEM) & $13.3(0.9)$ & | $3.5(1.2)$ & $9.6(1.1)$ \\
\hline Smoker & $9(50.0)$ & $7(38.9)$ & $4(33.3)$ \\
\hline Age of onset of MDD (year), mean (SEM) & $N / A$ & $46.4(4.7)$ & $44.83(6.8)$ \\
\hline Number (\%) of deaths due to suicide & 0 & $12(66.7)$ & $8(66.7)$ \\
\hline Family history of depression, number (\%) & 0 & 7 (38.8) & $2(16.7)$ \\
\hline
\end{tabular}

Abbreviations: AD, antidepressant drugs; MDD, major depressive disorder; n, number of subjects; N/A, not applicable; PMI (h), post-mortem interval in hours; SEM, standard error of the mean.

and two subjects had not taken ADs in the last 3 years before death. Three subjects were given prescribed antidepressants within the last 30 days before death; however, they were considered non-compliant (as indicated by the negative report in 'Toxicology'; Supplementary Table 1b). In addition, two of the prescribed antidepressants are primarily selective serotonin reuptake inhibitors (SSRIs), which target monoamine transporters (Tatsumi et al, 1997) and do not affect measures indicative of MAO A density (Meyer et al, 2009).

Additional information pertaining to individual illness characteristics is disclosed for subjects with MDD (Supplementary Table 1c; Table 1). A family history of depression was noted in $38.8 \%$ of the subjects with MDD (data not shown).

Twelve (12) MDD subjects treated with ADs. Age, gender, race, smoking status, PMI, $\mathrm{pH}$, freezer storage time, prescribed medication, toxicology reports, and additional information pertaining to individual illness characteristics are disclosed for MDD subjects treated with ADs (Table 1; Supplementary Table 1d). The average age (years, mean \pm SEM) of MDD subjects treated with antidepressants was $55.33 \pm 5.7$ and eight subjects were male. Eight subjects committed suicide (67\%); other causes of death included cardiovascular disease, hypertension, and cardiomyopathy (data not shown).

Postmortem blood or urine from the MDD subjects undergoing $\mathrm{AD}$ therapy revealed the presence of these drugs by toxicological screenings (none of which were MAO inhibitors - Supplementary Table 1d). Related family histories included depression and alcohol abuse; only $16.7 \%$ of the medicated MDD subjects had a family history of depression (data not shown). None of the subjects being pharmaceutically treated for MDD had implications of alcohol/drug abuse at or near their time of death. The individual illness characteristics for MDD subjects treated with ADs are shown in Supplementary Table le.

\section{Tissue Preparation}

Frozen tissues $(300 \mathrm{mg} / \mathrm{subject})$ from the prefrontal cortex were homogenized on ice in a $0.5 \mathrm{ml}$ solution containing $1 \mathrm{mM}$ EDTA, $10 \mathrm{mM}$ Tris-HCL ( $\mathrm{pH} 7)$, and fresh protease inhibitor. The homogenizing solution was placed at $-80^{\circ} \mathrm{C}$ for $20 \mathrm{~min}$, thawed and centrifuged at $4^{\circ} \mathrm{C}(2800-3000 \mathrm{rpm})$ for $10 \mathrm{~min}$. The supernatant was then stored at $-80^{\circ} \mathrm{C}$ until use. The protein concentrations of the homogenized samples were assessed using the BCA Protein Assay kit (Pierce).

\section{Western Blot}

A total of $50 \mu \mathrm{g}$ (per well) of total proteins were analyzed by $10.5 \%$ sodium dodecylsulfate-polyacrylamide gel electrophoresis and transferred to polyvinylidene fluoride membranes. After the transfer, membranes were blocked at room temperature for $1 \mathrm{~h}$ in milk-blocking buffer. The membranes were then incubated with rabbit anti-R1 antibody ( $1: 250$; Bethyl Laboratories) or mouse anti-MAO A antibody $\left(1: 250\right.$; Santa Cruz Biotechnology) overnight at $4{ }^{\circ} \mathrm{C}$. These antibodies specifically recognize human R1 $(\sim 56 \mathrm{kDa})$ and human MAO A $(\sim 61 \mathrm{kDa})$ proteins, respectively. The specificity of the anti-MAO A antibody was confirmed by a western blot analysis comparing MAO A protein detection in human brain tissues and human embryonic kidney cells that do not contain MAO A protein (Supplementary Figure 1). The membranes were then incubated with the respective secondary antibody, and 
subjected to chemiluminescent procedures (SuperSignal West Pico Chemiluminescent Substrate) and visualized with a Bio-Rad imaging system (Ou et al, 2009a; Ou et al, 2001).

An equal number of samples from the healthy control, MDD, and medicated MDD groups were immunoblotted on the same gel. Duplicate, independent preparations were also made and immunoblotted on separate gels. Protein bands were visualized by the Molecular Imager ChemiDoc XRS + System (Bio-Rad). For analysis, the band intensities for R1 and MAO A were calculated and normalized to the band intensities for $\beta$-actin using the Quantity One analysis software associated with the ChemiDoc System. Independently, a standard curve was established for R1 or MAO A using increasing concentrations of total protein from a human control subject. The optical densities for $\mathrm{R} 1$ and MAO A displayed a linear relationship relative to the total protein concentration (Supplementary Figure 2).

Expression levels of $\beta$-actin were also measured as described above using ChemiDoc System and analyzed by Quantity One software to establish loading controls. $\beta$-Actin is a reliable protein marker that is commonly used as a loading control for western blot experiments because it is very abundant in cells (Greer et al, 2010). $\beta$-Actin was obtained by stripping each membrane used for the determination of $\mathrm{R} 1$ or MAO A. The stripped blot was incubated overnight with anti- $\beta$-actin antibody (1:10000; MAB1501, Millipore) and was subsequently incubated with goat anti-mouse antibody IgG-HRP ( $1: 2500$, Santa Cruz Biotechnology). Therefore, the final protein levels for R1 or MAO A are normalized as a ratio of $\mathrm{R} 1 /$ actin or MAO A/actin (Dwivedi et al, 2003).

\section{MAO A Catalytic Activity Assay}

Brain tissue was used to determine MAO A catalytic (enzymatic) activity. After homogenization, $100 \mu \mathrm{g}$ of total protein of each sample were incubated with $100 \mu \mathrm{M}$ $\left[{ }^{14} \mathrm{C}\right]$ serotonin in the assay buffer $(50 \mathrm{mM}$ sodium phosphate buffer, $\mathrm{pH} \mathrm{7.4)}$ at $37^{\circ} \mathrm{C}$ for $20 \mathrm{~min}$, and the reaction was terminated by the addition of $100 \mu \mathrm{l}$ of $6 \mathrm{~N} \mathrm{HCl}$. Subsequently, the reaction products were extracted with benzene/ethyl acetate $(1: 1)$ and centrifuged at room temperature for $7 \mathrm{~min}$. The organic phase containing the reaction product with radioactivity was transferred to a scintillation vial and $5 \mathrm{ml}$ of scintillation fluid was added to the vial (Beckman Coulter Ready Safe Liquid Scintillation Cocktail). Liquid scintillation spectroscopy was then used to determine its radioactivity (Ou et al, 2006a).

Supplementary Figure 3 demonstrates the reliability of the MAO A assay involving frozen postmortem human tissues as linear responses were established as a function of protein concentration. An additional study (Supplementary Figure 4) also indicates that there is no significant difference in MAO A catalytic activities of mice brain specimens with different frozen storage times, which further suggests the feasibility and reliability of this assay for our current study with human subjects. Furthermore, the specificity of the MAO A assay related to postmortem brain tissue was evaluated using different concentrations of an MAO A inhibitor (M30) with fresh mice cortex as a positive control and human platelets as a negative control
(Supplementary Figure 5). These additional data suggest that the assessment of MAO A catalytic activity in the current study with human postmortem brain tissue is specific and reliable.

\section{Statistical Analysis}

One-way ANOVA and Dunnett adjusted tests were used for comparing the three groups (healthy controls, untreated MDD, and MDD with ADs). The data are reported as mean $\pm S E M$, and a value of $p<0.05$ was considered statistically significant. The potential influence of age, sex, race, smoking habit, $\mathrm{PMI}$, tissue $\mathrm{pH}$, tissue storage time on $\mathrm{R} 1$ and MAO A, duration of illness, number of depressive episodes, and suicide were examined by Student's $t$-test, $\chi^{2}$ tests, or Fisher's exact tests and no effect was detected in relation to these variables and protein expression levels. Multivariate linear regression analyses yielded results statistically similar to the independent $t$-tests (data not shown).

\section{RESULTS}

\section{R1 Was Significantly Decreased in Untreated MDD Subjects and MDD Subjects Undergoing AD Therapy Compared with Healthy Controls}

We determined whether R1 levels would be lower in autopsied brains from untreated and treated MDD subjects compared with psychiatrically normal control subjects based on the observation that $\mathrm{R} 1$ is decreased in synthetically induced cellular stress in human neuroblastoma cells (Chen et al, 2005; Ou et al, 2006b). Figure 1aA shows a representative western blot for the immunolabeling of R1 in individual prefrontal cortex specimens from three psychiatrically normal control subjects (healthy controls), three MDD subjects, and three antidepressant-treated MDD $(\mathrm{MDD}+\mathrm{AD})$ subjects. Statistical analysis of the western blotting by the global ANOVA revealed the differences among the three groups $\left(\mathrm{F}_{2,34}=5.77, p\right.$-value $\left.<0.01\right)$. The Dunnet's post hoc procedure identified a significant difference between healthy control group and MDD group $($ diff $=1.00,95 \%$ CI $(0.29,1.70) ; p<0.005)$. We also found a significant difference between healthy control group and $\mathrm{MDD}+\mathrm{AD}$ group $(\operatorname{diff}=0.80,95 \% \mathrm{CI}(0.02,1.59) ; p<0.05)$, and the difference is of a similar magnitude for both of the $\mathrm{MDD}$ and $\mathrm{MDD}+\mathrm{AD}$ groups compared with healthy control group (Figure 1a, columns MDD + AD vs MDD). The R1/actin ratio was $2.64 \pm 0.28$ (mean \pm SEM, $N=18$ ) for healthy control subjects, $1.65 \pm 0.18$ (mean \pm SEM, $N=18$ ) for untreated MDD subjects, and $1.84 \pm 0.19$ (mean $\pm \mathrm{SEM}, N=12$ ) for MDD subjects treated with ADs (Figure $1 \mathrm{aB}$ ), while no change was detected for $\beta$-actin levels. Therefore, R1 was significantly decreased in untreated MDD subjects by 37.5 and $30.3 \%$ for medicated MDD subjects in comparison with healthy control subjects, respectively. Based on this statistical analysis, $\mathrm{R} 1$ levels were not significantly correlated with age, sex, race, smoking status, PMI, storage time (Table 2), or features of MDD (duration of illness and number/duration of episodes; data not shown). 


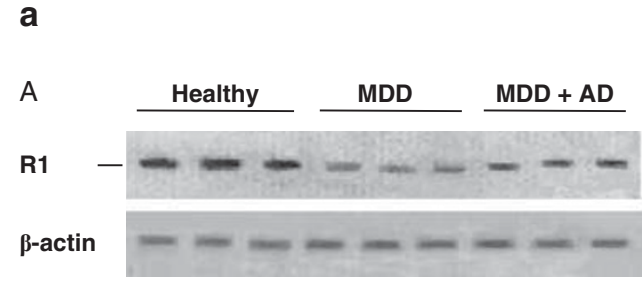

b

A

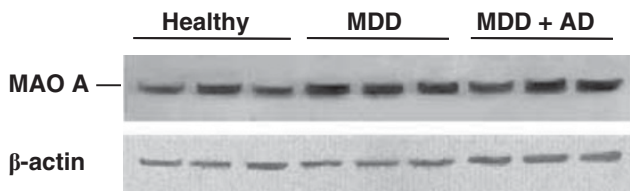

$\beta$-actin
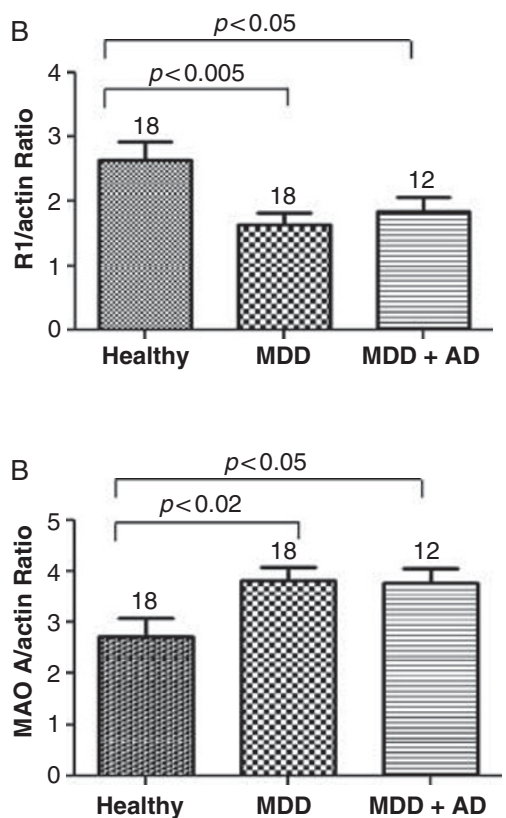

C

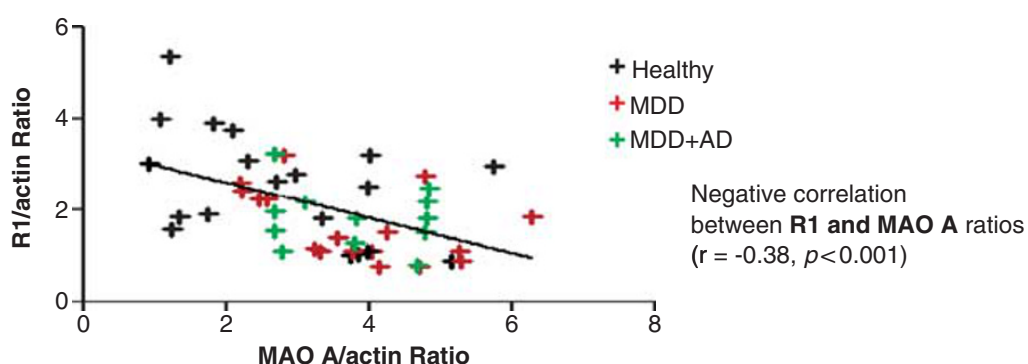

Figure I Quantitative analysis of protein expression was examined by western blot analysis. (a, b) Protein expression levels of RI and MAO A in the postmortem prefrontal cortex of I 8 psychiatrically normal control (Healthy) subjects, 18 subjects with MDD and I2 MDD subjects treated with ADs (MDD + AD). Representative western blots showing the immunolabeling of RI (aA), MAO A (bA) and $\beta$-actin in individual postmortem prefrontal cortex specimens of three healthy control subjects, three subjects with MDD, and three antidepressant-treated MDD subjects. (aB) RI expression is plotted for individual subjects with the mean \pm SEM represented by the long and two short horizontal lines, respectively. Each RI protein band was evaluated from two independent preparations by measuring its relative intensity and normalizing that value to the density of $\beta$-actin. (bB) MAO A protein levels in the prefrontal cortex of healthy controls, untreated and treated MDD subjects were plotted for individual subjects with the mean \pm SEM represented by the long and two short horizontal lines, respectively. Each MAO A protein band was evaluated from two independent preparations by measuring its relative intensity and normalized to the density of $\beta$-actin. (c) Graphic representation of the negative correlation between RI and MAO A levels in psychiatrically normal control subjects (black), subjects with MDD (red), and MDD subjects treated with AD therapy (green).

\section{MAO A Protein Levels Are Significantly Elevated in Untreated MDD Subjects and MDD Subjects with Antidepressant Treatment as Compared with Healthy Controls}

In parallel with the reduction of the MAO A-repressor, R1, we also determined the protein levels of MAO A in the prefrontal cortex of the depressed and psychiatrically normal control subjects. As expected, MAO A protein levels were significantly increased in MDD subjects (Figure 1b; $p<0.02$ ). Figure $1 \mathrm{bA}$ shows a representative western blot for the immunolabeling of MAO A in individual prefrontal cortex specimens from three psychiatrically normal control subjects (healthy controls), three MDD subjects, and three antidepressant-treated MDD subjects. Statistical analysis of the western blotting by the global ANOVA revealed differences among the three groups $\left(\mathrm{F}_{2,34}=4.03\right.$, $p$-value $<0.03)$. The Dunnet's post hoc procedure identified a significant difference between healthy control group and MDD group $(\operatorname{diff}=1.09,95 \% \mathrm{CI}(0.11,2.07) ; p<0.02)$. We also found a significant difference between healthy control group and $\mathrm{MDD}+\mathrm{AD}$ group ( $\mathrm{diff}=1.052,95 \% \mathrm{CI}(0.02$, $1.86) ; p<0.05$ ), and the difference is of a similar magnitude for both MDD and MDD + AD groups compared with healthy control group (Figure $1 \mathrm{bB}$, columns MDD + AD vs MDD). The MAO A/actin ratio was $2.72 \pm 0.34$ (mean \pm SEM, $N=18)$ for the healthy control group, $3.82 \pm 0.27$ (mean \pm SEM, $N=18$ ) for the untreated depressed group, and $3.78 \pm 0.28$ (mean \pm SEM, $N=12$ ) for the MDD subjects treated with antidepressants. Therefore, the expression of MAO A was significantly increased by $40.4 \%$ in untreated MDD subjects and by $39 \%$ in medicated MDD subjects $(\mathrm{MDD}+\mathrm{AD})$, respectively. Similar to R1 levels, MAO A levels were not significantly correlated with age, sex, race, 
Table 2 Summary of Statistic Analyses Among Psychiatrically Normal Control Subjects (Healthy), Subjects with Major Depressive Disorder (MDD) and MDD Subjects with Antidepressant Drugs (MDD+AD)

\begin{tabular}{|c|c|c|c|c|c|}
\hline & Healthy $(n=18)$ & MDD $(n=18)$ & MDD+AD $(n=12)$ & Statistic $^{a}$ & $p$-value \\
\hline Age & $49.61 \pm 3.36$ & $54.61 \pm 4.66$ & $55.33 \pm 5.65$ & 0.50 & 0.6100 \\
\hline Gender (\% male) & $61.1 \%$ & $66.7 \%$ & $66.7 \%$ & 0.15 & 0.9269 \\
\hline PMI & $20.04 \pm 1.87$ & $20.66 \pm 1.64$ & $21.03 \pm 2.08$ & 0.07 & 0.9334 \\
\hline $\mathrm{pH}$ & $6.60 \pm 0.080$ & $6.56 \pm 0.066$ & $6.58 \pm 0.074$ & 0.07 & 0.9339 \\
\hline $\mathrm{RI} /$ actin & $2.64 \pm 0.28$ & $1.65 \pm 0.18$ & $1.84 \pm 0.19$ & 5.77 & 0.0059 \\
\hline MAO A/actin & $2.72 \pm 0.34$ & $3.82 \pm 0.27$ & $3.78 \pm 0.28$ & 4.03 & 0.0202 \\
\hline
\end{tabular}

${ }^{a}$ Continuous data compared with F-statistic with 2 (num) and 34 (den) df categorical data compared with $\chi^{2}$ test with I df.

${ }^{\mathrm{b}} \mathrm{AA}$, African American.

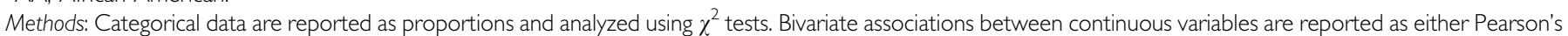

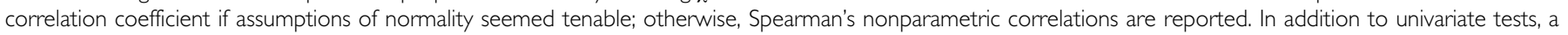
multivariate model was used to compare the RI/actin ratio between MDD and healthy controls or between MDD+AD and healthy controls, while adjusting for potentially confounding factors. Similar tests were also performed for the MAO A outcomes. We considered $P<0.05$ evidence of statistical significance.

I. General outcomes

There are no significant differences in demographic variables (age, gender, race, $\mathrm{PMI}, \mathrm{pH}$, and smoker) between the groups. There is a difference in storage time; however, after using a multivariate linear regression model, adjusting for storage time, there is a significant difference between MDD and controls and between MDD+AD and controls for both RI and MAO A as described below.

2. The detailed analysis for RI

There is not a significant correlation between the RI/actin ratio and $\mathrm{PMI}(r=0.08, p$-value $=0.6085)$, storage time $(r=-0.02, p$-value $=0.9 \mathrm{I} 30)$, $\mathrm{pH}(r=0.23$, $p$-value $=0.1112)$, or age $(r=-0.23$, $p$-value $=0.1157)$.

The RI/actin ratio is not significantly different for race $(t=1.61, \mathrm{df}=46, p$-value $=0.1 \mid 42)$, gender $(t=-0.16, \mathrm{df}=46, p$-value $=0.87 \mid 5)$, or smoking status $(t=-1.21, \mathrm{df}=46, p$-value $=0.2317)$.

In a multivariate linear regression model, adjusting for age, race, gender, $\mathrm{pH}$, storage time, and PMI, the difference between groups remains significant for $\mathrm{RI}$

$\left(F_{2,38}=4.7 \mathrm{I}, \mathrm{p}\right.$-value $\left.=0.0 \mathrm{I} 48\right)$. Dunnett's post hoc procedure identifies differences between $\mathrm{MDD}$ and control subjects (diff $\left.=1.00,95 \% \mathrm{Cl}(0.3 \mathrm{I}, \mathrm{I} .69)\right)$ and also between MDD+AD and controls (diff $=0.93,95 \% \mathrm{Cl}(0.14,1.72)$ ).

3. The detailed analysis for MAO A

There is not a significant correlation between the MAO A/actin ratio and $\mathrm{PMI}(r=-0.10$, $p$-value $=0.4994)$, storage time $(r=-0.13$, $p$-value $=0.4003)$, $\mathrm{pH}$ $(r=-0.10$, $p$-value $=0.4899)$, or age $(r=0.01$, $p$-value $=0.9292)$.

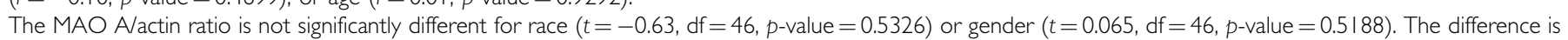
also not significant for smoking status $(t=0.49, \mathrm{df}=46, p$-value $=0.6272)$.

In a multivariate linear regression model, adjusting for age, race, gender, $\mathrm{pH}$, storage time, and $\mathrm{PMI}$, the difference between groups remains significant for $\mathrm{MAO} A$ $\left(F_{2,38}=3.50, p\right.$-value $\left.=0.0402\right)$. Dunnett's post hoc procedure identifies differences between $\mathrm{MDD}$ and control subjects $(\mathrm{diff}=1.09,95 \% \mathrm{Cl}(0.08,2.10))$ and also between MDD+AD and controls (diff $=1.12,95 \% \mathrm{Cl}(0.07,2.38)$ ).

smoking status, PMI, storage time (Table 2), or features of MDD (duration of illness and number/duration of episodes; data not shown).

\section{Reduction in R1 Protein Is Negatively Correlated with the Significant Increase in MAO A Protein Levels}

A correlation analysis was performed between R1/actin and MAO A/actin expression levels in each subject because $\mathrm{R} 1$ is a transcriptional repressor of MAO A gene expression. As expected, the result shows a highly significant negative correlation between R1/actin and MAO A/actin (as R1 levels decrease, MAO A protein expression increase; MAO A Pearson's $r=-0.385, p=0.0005$; Figure 1c) when all three groups (healthy control subjects, untreated MDD subjects, and MDD subjects undergoing AD therapy) were combined together. Combining only the subjects with MDD (untreated and treated with ADs), R1 protein expression also exhibited a significant negative correlation with $\mathrm{MAO}$ A protein levels $(r=-0.24, p=0.048$; Supplementary Figure 6$)$.
Additionally, a multivariate analysis showed that R1 and MAO A levels are not significantly different for gender, race, PMI, and storage time (Table 2). Features of MDD such as duration of illness and number/duration of episodes did not exhibit any correlation with R1 or MAO A protein expression levels. Similarly, R1 and MAO A levels did not correlate with smoking status (possibly due to mild nicotine exposure (Beck et al, 1981), which is consistent with a previous report in regards to the effect of long-term cigarette smoking on the human locus coeruleus (Klimek et al, 2001)). Other studies by Fowler et al (1996) and Leroy et al (2009) show that MAO A levels are decreased in the brains of individuals with chronic, excessive nicotine exposure; however, in the current study, the magnitude of cigarette smoking in most of the subjects was considered to be mild (one pack or less per day; data not shown) in comparison to the Fowler and Leroy study. For the relation of R1 or MAO A with suicide, the statistical analysis showed no differences in the protein expression of $\mathrm{R} 1$ or MAO A between suicide and non-suicide groups (Supplementary Table 2). 
With regard to past antidepressant treatment among the 18 untreated MDD subjects, 13 of these MDD subjects had never been treated with antidepressants. We have also compared the protein levels of R1 and MAO A in these 13 MDD subjects with those of 18 healthy control subjects. The result showed that, like the entire group of MDD subjects $(n=18), \mathrm{R} 1$ levels were significantly decreased (the average R1/actin ratio: $1.516 \pm 0.21$, while MAO A protein expression was significantly increased (the average MAO A/actin ratio: $3.84 \pm 0.23$ ) in these $13 \mathrm{MDD}$ subjects who had no antidepressant history compared with those in 18 healthy control subjects; furthermore, the differences are even slightly more robust in these 13 medication-free MDD subjects than those in the entire untreated MDD group (R1/ actin ratio: $1.65 \pm 0.18$ and MAO A/actin ratio: $3.82 \pm 0.27$ ). Therefore, the past antidepressant treatment in some MDD subjects did not affect the current findings.

\section{MAO A Enzymatic Activity Is Significantly Increased in Both Untreated MDD Subjects and Subjects Treated with ADs}

Catalytic (enzymatic) activity of MAO A was also assessed in the prefrontal cortex revealing that MAO A enzymatic activity was comparably increased in MDD subjects treated with ADs by $19 \%(p$-value $<0.04)$, as in untreated MDD subjects by $24.5 \%$ ( $p$-value $<0.05)$, when compared with healthy control subjects as shown in Figure 2. The global ANOVA found differences among the three groups $\left(\mathrm{F}_{2,6}=6.118, p\right.$-value $\left.<0.0356\right)$ with no statistically significant difference between the two depressed groups. In this statistical analysis, levels of MAO A enzymatic activity were not significantly correlated with age, sex, race, suicide,

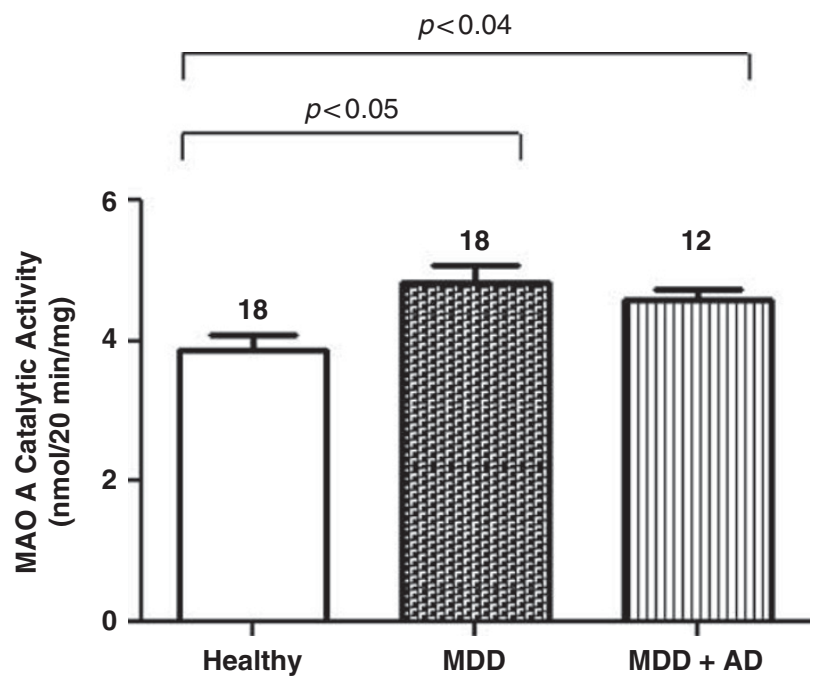

Figure 2 MAO A catalytic (enzymatic) activity levels in the postmortem prefrontal cortex of psychiatrically normal control subjects (Healthy), subjects with MDD and MDD subjects treated with ADs (MDD + AD). The enzymatic activity of MAO A from four independent preparations was determined by MAO A catalytic activity assay. The average catalytic activities are graphically shown for 18 healthy control subjects, 18 subjects with MDD, and 12 MDD subjects treated with ADs. Each bar represents the mean MAO A catalytic activity for the group and the SEM is indicated by the horizontal line. smoking status, PMI, storage time, or features of MDD (duration of illness and number/duration of episodes). This result is consistent with a previous report (Sherif et al, 1991), in which the MAO A catalytic activity was significantly increased in the human postmortem hypothalamic region of suicide victims with depressive disorders compared with psychiatrically normal control subjects.

\section{DISCUSSION}

This study provides greater insight into the mechanisms involved in the manifestation of MDD by examining R1 protein levels in human postmortem brain tissues for the first time. The protein expression levels of R1 and MAO A were examined by western blot analysis in the prefrontal cortex of three groups of subjects: (1) MDD with no antidepressant medications, (2) MDD with antidepressant medications, and (3) age- and gender-matched healthy controls. R1 levels were significantly decreased by $37.5 \%$ in untreated MDD subjects, whereas MAO A levels were significantly increased by $40 \%$. R1 levels in depressed subjects taking medications at the time of death were similarly decreased compared with non-medicated depressed subjects, demonstrating that current antidepressants do not increase R1 protein expression. These findings may have major implications for (1) identifying the mechanism underlying the manifestation of greater MAO A levels in MDD; (2) identifying a mechanism to explain the cell loss associated with MDD; and (3) identify a new target (R1) of drug therapy for the treatment of MDD.

$\mathrm{R} 1$ has been identified as an upstream repressor of MAO A (Chen et al, 2005), and the roles of R1 and MAO A in cell growth and the apoptotic signaling pathway have been previously examined as well. After serum starvationinduced apoptosis in SK-N-BE(2)-C cells, R1 expression decreased, whereas MAO A expression increased. Furthermore, the over-expression of R1 (or pharmaceutical inhibition of MAO A) prevents apoptosis and decreases MAO A activity, suggesting that the function of $\mathrm{R} 1$ in cell growth may be mediated by the inhibition of MAO A activity (Ou et al, 2006b). Our current study shows that this reduction of $\mathrm{R} 1$ expression may contribute to increased MAO A levels in individuals with MDD. Therefore, this study is fundamental to the development of novel therapeutic strategies because $\mathrm{R} 1$ protein expression is inversely correlated with MAO A protein levels in MDD subjects. The MAO A enzyme activity levels were also significantly increased in MDD subjects compared with those in healthy control subjects, although the increase in enzyme activity levels (by $24.5 \%$ ) was less than the increase in MAO A protein levels (by 40.4\%); this may be due to a preferential effect of PMI to reduce enzyme activity. Given the role of $\mathrm{R} 1$ in apoptotic cell death, drugs that directly target this MAO A-repressor should be investigated because R1 not only inhibits MAO A gene expression (Chen et al, 2005) but also enhances cell growth and prevents apoptosis (Huang et al, 2005; Ou et al, 2006b). A new drug that targets R1 may have more beneficial effects than drugs that only target MAO A.

Moreover, the manipulation of R1 by compounds that will increase R1 levels in depression may (1) overcome excessive 
MAO A synthesis associated with current treatment resistance; (2) prevent the recurrence of depressive episodes because commonly prescribed drugs (such as SSRIs) neither inhibit MAO A levels nor specifically target R1 (Meyer et al, 2009); (3) reduce MAO A-mediated apoptotic cell death, increase neuroprotection, and promote neuroplasticity; maximizing these effects is also relevant to the treatment of depressive disorders (Dwivedi et al, 2006; Sanacora, 2008). Subsequent studies should also address the application of R1 levels as a potential diagnostic marker for depressive disorders and treatment assessment by measuring blood levels of this protein in current and future patients.

An additional study by Thalmeier et al (2008) involving 11 suicide victims (seven of which had depression) analyzed the expression of over 23000 transcripts in the orbitofrontal cortex (Brodmann area 11), and identified R1 (also called CDCA7L) as one of nine more prominent transcripts associated with suicide. They reported that R1 mRNA levels were decreased significantly in suicide victims $(p \leqslant 0.01)$ as determined by quantitative real-time polymerase chain reaction. Interestingly, five of the depressed subjects in their study were being treated with $\mathrm{AD}$ therapy at the time of death as indicated by toxicological screenings (Thalmeier et al, 2008). With a hypothesis driven approach, our study is consistent with Thalmeier and demonstrates that R1 protein levels are strongly reduced (37.5\% lower) in MDD.

This study characterizes the possible pathways elucidating the role of R1-MAO A in the pathology of MDD. New insights into the monoamine theory of depression have come from PET studies revealing increased levels of MAO A in the prefrontal cortex (Meyer et al, 2006; Meyer et al, 2009); our current report of enhanced levels of MAO A is consistent with this research. MAO A levels of medication free, depressed individuals were assessed in a recent study by PET scans using $\left[{ }^{11} \mathrm{C}\right]$ harmine, a radiotracer for MAO A; the average MAO A binding was elevated by $34 \%$ in individuals with MDD (Meyer et al, 2006). A subsequent study replicated this finding and evaluated the relationship between MAO A binding and state of illness. MAO A binding was elevated in major depressive episodes prior to antidepressant treatment, remained elevated for 6 weeks after SSRI treatment (reflecting a resistant pathological process); and subjects in recovery from MDD had significantly higher prefrontal and anterior cingulate cortex MAO A binding, which was most prominent in those who subsequently had recurrence of their major depressive episodes (Meyer et al, 2009). Current treatment options neither achieve adequate remission rates nor adequately prevent recurrence (Kessler et al, 2003); as a result, identification of pathological molecular mechanisms resistant to common antidepressants is important to successfully treat MDD.

In summary, this study identified strongly diminished levels of R1, an upstream transcriptional repressor of MAO $A$, and highly elevated levels of MAO A in subjects with MDD. These results argue that, in MDD, diminished levels of R1 lead to an enhanced expression of MAO A. Our data also demonstrate that the treatment of depression with an SSRI will neither suppress MAO A levels, nor significantly enhance levels of $\mathrm{R} 1$ in the prefrontal cortex. Given the associations of lower R1 with reduced cell proliferation and increased apoptosis, lower R1 may be an important factor in the pathophysiology of depression; therefore, the development of new medications to restore brain levels of $\mathrm{R} 1$ and decrease MAO A levels may provide therapeutic benefits for patients with MDD.

\section{ACKNOWLEDGEMENTS}

This research was supported by Public Health Service Grants P20 RR 017701, MH67996, National Alliance for Research on Schizophrenia and Depression, the Canadian Institutes of Health Research, and an Intramural Research Support grant from the University of Mississippi Medical Center. We gratefully acknowledge the invaluable contributions made by the families consenting to donate brain tissue and to be interviewed. The kind assistance of the Cuyahoga County Coroner's office, Cleveland, Ohio, is also noted. We thank Dr Herbert Y Meltzer for psychiatric assessment assistance. Nicole Herbst, Timothy De Jong, Shawnnette Nelson, and Nicole Peak assisted with human tissues and obtaining written consent. Dr Gouri Mahajan, Chinelo Udemgba, and John Sullivan assisted with tissue preparation and western blot analyses.

\section{DISCLOSURE}

Dr Meyer has applied for a patent to apply MAO measures to diagnose or identify subtypes of MDD and he has had operating grant support/consultation with GlaxoSmith Kline, BristolMyersSquibb, Lundbeck, SK Life Sciences, and Eli-Lilly. All other authors declare no conflict of interest.

\section{REFERENCES}

Andreazza AC, Shao L, Wang JF, Young LT (2010). Mitochondrial complex I activity and oxidative damage to mitochondrial proteins in the prefrontal cortex of patients with bipolar disorder. Arch Gen Psychiatry 67: 360-368.

Bartholomeeusen K, De Rijck J, Busschots K, Desender L, Gijsbers $\mathrm{R}$, Emiliani $\mathrm{S}$ et al (2007). Differential interaction of HIV-1 integrase and JPO2 with the C terminus of LEDGF/p75. J Mol Biol 372: 407-421.

Beck GJ, Doyle CA, Schachter EN (1981). Smoking and lung function. Am Rev Respir Dis 123: 149-155.

Cases O, Seif I, Grimsby J, Gaspar P, Chen K, Pournin S et al (1995). Aggressive behavior and altered amounts of brain serotonin and norepinephrine in mice lacking MAOA. Science (New York, NY) 268: 1763-1766.

Chen K, Ou XM, Chen G, Choi SH, Shih JC (2005). R1, a novel repressor of the human monoamine oxidase A. J Biol Chem 280: 11552-11559.

Chen K, Ou XM, Wu JB, Shih JC (2011). Transcription factor E2Fassociated phosphoprotein (EAPP), RAM2/CDCA7L/JPO2 (R1), and simian virus 40 promoter factor 1 (Sp1) cooperatively regulate glucocorticoid activation of monoamine oxidase B. $\mathrm{Mol}$ Pharmacol 79: 308-317.

Chouinard G, Young SN, Annable L (1985). A controlled clinical trial of L-tryptophan in acute mania. Biol Psychiatry 20: 546-557.

De Zutter GS, Davis RJ (2001). Pro-apoptotic gene expression mediated by the p38 mitogen-activated protein kinase signal transduction pathway. Proc Natl Acad Sci USA 98: 6168-6173. 
Deep-Soboslay A, Akil M, Martin CE, Bigelow LB, Herman MM, Hyde TM et al (2005). Reliability of psychiatric diagnosis in postmortem research. Biol Psychiatry 57: 96-101.

Dejong TM, Overholser JC (2009). Assessment of depression and suicidal actions: agreement between suicide attempters and informant reports. Suicide Life Threat Behav 39: 38-46.

Du L, Bakish D, Ravindran A, Hrdina PD (2004). MAO-A gene polymorphisms are associated with major depression and sleep disturbance in males. Neuroreport 15: 2097-2101.

Du L, Faludi G, Palkovits M, Sotonyi P, Bakish D, Hrdina PD (2002). High activity-related allele of MAO-A gene associated with depressed suicide in males. Neuroreport 13: 1195-1198.

Duman RS, Monteggia LM (2006). A neurotrophic model for stress-related mood disorders. Biol Psychiatry 59: 1116-1127.

Dwivedi Y, Mondal AC, Rizavi HS, Faludi G, Palkovits M, Sarosi A et al (2006). Differential and brain region-specific regulation of Rap-1 and Epac in depressed suicide victims. Arch Gen Psychiatry 63: 639-648.

Dwivedi Y, Rizavi HS, Conley RR, Roberts RC, Tamminga CA, Pandey GN (2003). Altered gene expression of brain-derived neurotrophic factor and receptor tyrosine kinase B in postmortem brain of suicide subjects. Arch Gen Psychiatry 60: 804-815.

Dwivedi Y, Rizavi HS, Zhang H, Mondal AC, Roberts RC, Conley $\mathrm{RR}$ et al (2009). Neurotrophin receptor activation and expression in human postmortem brain: effect of suicide. Biol Psychiatry 65: 319-328.

Egede LE (2004). Diabetes, major depression, and functional disability among U.S. adults. Diabetes Care 27: 421-428.

Fan M, Liu B, Jiang T, Jiang X, Zhao H, Zhang J (2010). Metaanalysis of the association between the monoamine oxidase- $A$ gene and mood disorders. Psychiatr Genet 20: 1-7.

First MBSR, Gibbon M, Williams JBW (1996). Structured Clinical Interview for DSM-IV Axis I Disorders - Patient Edition, Version 2.0 ed. Biometrics Research Department, New York State Psychiatric Institute: New York.

Fowler JS, Volkow ND, Wang GJ, Pappas N, Logan J, Shea C et al (1996). Brain monoamine oxidase A inhibition in cigarette smokers. Proc Natl Acad Sci USA 93: 14065-14069.

Gawryluk JW, Wang JF, Andreazza AC, Shao L, Young LT (2010). Decreased levels of glutathione, the major brain antioxidant, in post-mortem prefrontal cortex from patients with psychiatric disorders. Int J Neuropsychopharmacol 14: 1-8.

Goridis C, Neff NH (1971). Monoamine oxidase in sympathetic nerves: a transmitter specific enzyme type. Br J Pharmacol 43: 814-818.

Greer S, Honeywell R, Geletu M, Arulanandam R, Raptis L (2010). Housekeeping genes; expression levels may change with density of cultured cells. J Immunol Meth 355: 76-79.

Huang A, Ho CS, Ponzielli R, Barsyte-Lovejoy D, Bouffet E, Picard $\mathrm{D}$ et al (2005). Identification of a novel c-Myc protein interactor, JPO2, with transforming activity in medulloblastoma cells. Cancer Res 65: 5607-5619.

Kang HJ, Adams DH, Simen A, Simen BB, Rajkowska G, Stockmeier CA et al (2007). Gene expression profiling in postmortem prefrontal cortex of major depressive disorder. J Neurosci 27: 13329-13340.

Keller MB, Boland RJ (1998). Implications of failing to achieve successful long-term maintenance treatment of recurrent unipolar major depression. Biol Psychiatry 44: 348-360.

Kelly TM, Mann JJ (1996). Validity of DSM-III-R diagnosis by psychological autopsy: a comparison with clinician ante-mortem diagnosis. Acta Psychiatrica Scandinavica 94: 337-343.

Kessler RC, Berglund P, Demler O, Jin R, Koretz D, Merikangas KR et al (2003). The epidemiology of major depressive disorder: results from the National Comorbidity Survey Replication (NCS-R). JAMA 289: 3095-3105.

Klimek V, Zhu MY, Dilley G, Konick L, Overholser JC, Meltzer HY et al (2001). Effects of long-term cigarette smoking on the human locus coeruleus. Arch Gen Psychiatry 58: 821-827.
Kunduzova OR, Bianchi P, Parini A, Cambon C (2002). Hydrogen peroxide production by monoamine oxidase during ischemia/ reperfusion. Eur J Pharmacol 448: 225-230.

Leroy C, Bragulat V, Berlin I, Gregoire MC, Bottlaender M, Roumenov D et al (2009). Cerebral monoamine oxidase A inhibition in tobacco smokers confirmed with PET and [11C]befloxatone. J Clin Psychopharmacol 29: 86-88.

Maertens GN, Cherepanov P, Engelman A (2006). Transcriptional co-activator p75 binds and tethers the Myc-interacting protein JPO2 to chromatin. J Cell Sci 119: 2563-2571.

Meyer JH, Ginovart N, Boovariwala A, Sagrati S, Hussey D, Garcia A et al (2006). Elevated monoamine oxidase a levels in the brain: an explanation for the monoamine imbalance of major depression. Arch Gen Psychiatry 63: 1209-1216.

Meyer JH, Wilson AA, Sagrati S, Miler L, Rusjan P, Bloomfield PM et al (2009). Brain monoamine oxidase A binding in major depressive disorder: relationship to selective serotonin reuptake inhibitor treatment, recovery, and recurrence. Arch Gen Psychiatry 66: 1304-1312.

Morilak DA, Frazer A (2004). Antidepressants and brain monoaminergic systems: a dimensional approach to understanding their behavioural effects in depression and anxiety disorders. Int J Neuropsychopharmacol 7: 193-218.

Ou XM, Chen K, Shih JC (2006a). Glucocorticoid and androgen activation of monoamine oxidase $\mathrm{A}$ is regulated differently by $\mathrm{R} 1$ and Sp1. J Biol Chem 281: 21512-21525.

Ou XM, Chen K, Shih JC (2006b). Monoamine oxidase A and repressor R1 are involved in apoptotic signaling pathway. Proc Natl Acad Sci USA 103: 10923-10928.

Ou XM, Lu D, Johnson C, Chen K, Youdim MB, Rajkowska G et al (2009a). Glyceraldehyde-3-phosphate dehydrogenase-monoamine oxidase B-mediated cell death-induced by ethanol is prevented by rasagiline and 1-R-aminoindan. Neurotox Res 16: $148-159$.

Ou XM, Stockmeier CA, Meltzer HY, Overholser JC, Jurjus GJ, Dieter L et al (2009b). A novel role for glyceraldehyde-3phosphate dehydrogenase and monoamine oxidase $B$ cascade in ethanol-induced cellular damage. Biol Psychiatry 67: $855-863$

Ou XM, Storring JM, Kushwaha N, Albert PR (2001). Heterodimerization of mineralocorticoid and glucocorticoid receptors at a novel negative response element of the 5-HT1A receptor gene. J Biol Chem 276: 14299-14307.

Rajkowska G, Miguel-Hidalgo JJ, Dubey P, Stockmeier CA, Krishnan KR (2005). Prominent reduction in pyramidal neurons density in the orbitofrontal cortex of elderly depressed patients. Biol Psychiatry 58: 297-306.

Reilly JG, McTavish SF, Young AH (1997). Rapid depletion of plasma tryptophan: a review of studies and experimental methodology. J Psychopharmacol 11: 381-392.

Sacher J, Wilson AA, Houle S, Rusjan P, Hassan S, Bloomfield PM et al (2010). Elevated brain monoamine oxidase A binding in the early postpartum period. Arch Gen Psychiatry 67: 468-474.

Sanacora G (2008). New understanding of mechanisms of action of bipolar medications. J Clin Psychiatry 69(Suppl 5): 22-27.

Schmidt HD, Duman RS (2007). The role of neurotrophic factors in adult hippocampal neurogenesis, antidepressant treatments and animal models of depressive-like behavior. Behav Pharmacol 18: 391-418.

Sherif F, Marcusson J, Oreland L (1991). Brain gamma-aminobutyrate transaminase and monoamine oxidase activities in suicide victims. Eur Arch Psychiatry Clin Neurosci 241: 139-144.

Shih JC (1991). Molecular basis of human MAO A and B. Neuropsychopharmacology 4: 1-7.

Shih JC, Chen K (1999). MAO-A and -B gene knock-out mice exhibit distinctly different behavior. Neurobiology $(B p) 7$ : 235-246. 
The MAO A-repressor, $R I$, in depressive disorder $\mathrm{S}$ Johnson et al

Shih JC, Grimsby J, Chen K, Zhu QS (1993). Structure and promoter organization of the human monoamine oxidase $A$ and B genes. J Psychiatry Neurosci 18: 25-32.

Stockmeier CA, Howley E, Shi X, Sobanska A, Clarke G, Friedman L et al (2009). Antagonist but not agonist labeling of serotonin$1 \mathrm{~A}$ receptors is decreased in major depressive disorder. J Psychiatr Res 43: 887-894.

Tatsumi M, Groshan K, Blakely RD, Richelson E (1997). Pharmacological profile of antidepressants and related compounds at human monoamine transporters. Eur J Pharmacol 340: 249-258.

Thalmeier A, Dickmann M, Giegling I, Schneider B, A MH, Maurer $\mathrm{K}$ et al (2008). Gene expression profiling of post-mortem orbitofrontal cortex in violent suicide victims. Int J Neuropsychopharmacol 11: 217-228.
Youdim MB, Bakhle YS (2006). Monoamine oxidase: isoforms and inhibitors in Parkinson's disease and depressive illness. $\mathrm{Br} \mathrm{J}$ Pharmacol 147(Suppl 1): S287-S296.

Youdim MB, Edmondson D, Tipton KF (2006). The therapeutic potential of monoamine oxidase inhibitors. Nat Rev 7: 295-309. Youdim MB, Fridkin M, Zheng H (2004). Novel bifunctional drugs targeting monoamine oxidase inhibition and iron chelation as an approach to neuroprotection in Parkinson's disease and other neurodegenerative diseases. J Neural Transm 111: 1455-1471.

Young SN, Smith SE, Pihl RO, Ervin FR (1985). Tryptophan depletion causes a rapid lowering of mood in normal males. Psychopharmacology (Berl) 87: 173-177.

Zhu QS, Chen K, Shih JC (1994). Bidirectional promoter of human monoamine oxidase A (MAO A) controlled by transcription factor Sp1. J Neurosci 14: 7393-7403.

Supplementary Information accompanies the paper on the Neuropsychopharmacology website (http://www.nature.com/npp) 